\title{
Observation of a Localization Transition in Quasiperiodic Photonic Lattices
}

\author{
Y. Lahini, ${ }^{1, *}$ R. Pugatch, ${ }^{1}$ F. Pozzi, ${ }^{2}$ M. Sorel, ${ }^{2}$ R. Morandotti, ${ }^{3}$ N. Davidson, ${ }^{1}$ and Y. Silberberg ${ }^{1}$ \\ ${ }^{1}$ Department of Physics of Complex Systems, The Weizmann Institute of Science, Rehovot, Israel \\ ${ }^{2}$ Department of Electrical and Electronic Engineering, University of Glasgow, Glasgow, Scotland \\ ${ }^{3}$ Institut National de la Recherche Scientifique, Université du Québec, Varennes, Québec, Canada
}

(Received 9 October 2008; published 30 June 2009)

\begin{abstract}
We report the observation of the signature of a localization phase transition for light in one-dimensional quasiperiodic photonic lattices, by directly measuring wave transport inside the lattice. Below the predicted transition point an initially narrow wave packet expands as it propagates, while above the transition expansion is fully suppressed. In addition, we measure the effect of focusing nonlinear interaction on the propagation and find it increases the width of the localized wave packets.
\end{abstract}

DOI: 10.1103/PhysRevLett.103.013901

The localization of waves in nonperiodic media is a universal phenomenon, occurring in a variety of different quantum and classical systems, including condensedmatter [1,2], Bose-Einstein condensates in optical potentials [3,4], quantum chaotic systems [5-7], sound waves [8], and light [9-19]. In disordered systems, a localization phase transition is expected to occur in three dimensions as the strength of disorder crosses a critical value $[1,2,14-16]$. Lower dimensional systems with uncorrelated disorder do not exhibit a localization phase transition [2]; however, finite size systems can exhibit a crossover from an extended to a localized phase, as was recently observed [17-19]. In 1979 Aubry and André predicted that for a certain class of quasiperiodic potentials, a localization phase transition can occur already in one dimension [20]. Here we report an experiment that realizes the AubryAndré (AA) model in quasiperiodic photonic lattices. We observe the signature of a localization phase transition by directly measuring the expansion rates of initially narrow wave packets propagating in the lattice. Below the transition all the modes of the system are extended and therefore an initially narrow wave packet eventually spreads across the entire lattice. Above the critical point, all modes are localized and expansion is suppressed [21]. In addition, we study the effect of weak nonlinear interactions below and above the transition.

The AA model describes a 1D tight-binding periodic lattice with on-site modulation, such that the ratio between the modulation and the lattice period is $\chi$ [20]:

$$
i \frac{\partial \psi_{n}}{\partial t}+\left[\beta_{0}+\lambda \cos (2 \pi n \chi)\right] \psi_{n}+C\left(\psi_{n-1}+\psi_{n+1}\right)=0
$$

where $\psi_{n}$ is the wave function at site $n, \beta_{0}$ is the single-site energy of the (unperturbed) periodic lattice, and $C$ is the tunneling rate between sites. It has been shown that if $\chi$ is an irrational Diophantine number [22], a sharp localization phase transition takes place at a modulation strength $\lambda=2$ (At this point, the Aubry-André Hamiltonian is self-dual [23] and coincides with the Harper Hamiltonian [24] that
PACS numbers: 42.25.Dd, 42.65.Tg, 61.44.Fw, 72.15.Rn

exhibits the famous Hofstadter butterfly fractal spectrum). In Fig. 1(a) we plot the amplitude of the ground state as a function of $\lambda / C$, calculated using Eq. (1) for a system with 99 sites and $\chi=(\sqrt{5}+1) / 2$ (the golden mean, which is a Diophantine number). At $\lambda / C=0$ the lattice is periodic and the ground state is extended over the entire lattice. As $\lambda$ is increased the ground state becomes rugged, but remains extended. Right above $\lambda / C=2$ the ground state
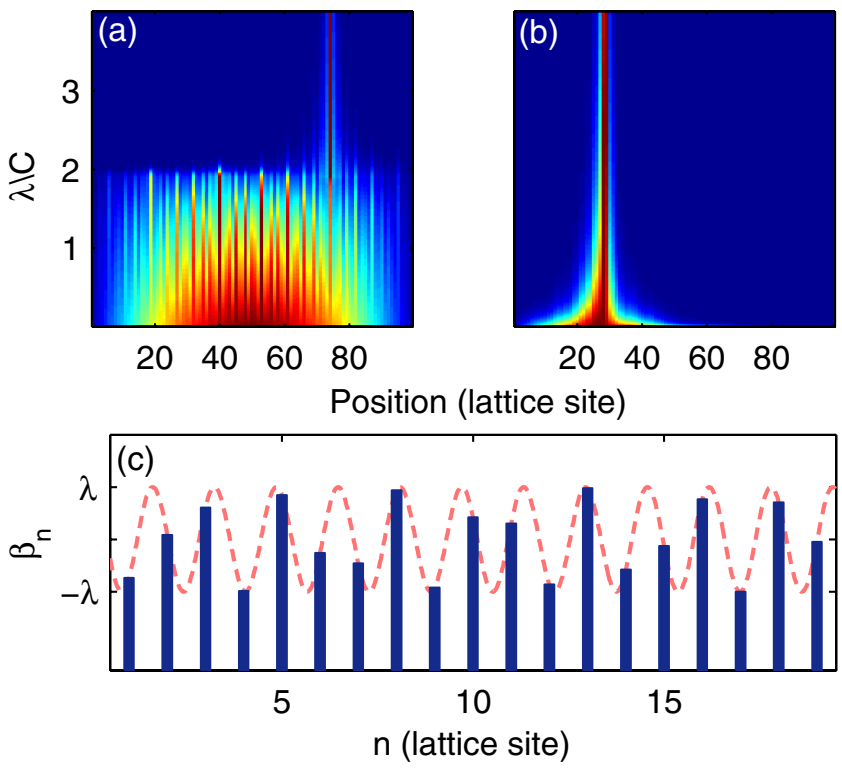

FIG. 1 (color online). (a) Calculated amplitude profile of the ground state of an incommensurate lattice (horizontal axis) versus the strength of the incommensurate modulation depth $\lambda$ (vertical axis). A sharp localization transition is observed at $\lambda=$ $2 C$. The wave function is extended for $\lambda / C<2$, and localized to a single site for $\lambda / C>2$. A similar transition occurs for all other eigenstates of the system. (b) The same calculation for a disordered lattice. Here the localization length goes continuously to zero. All horizontal cross sections are normalized to a unit maximum. (c) A quasiperiodic lattice is obtained by modulating the on-site eigenvalues of the lattice sites (bars, $\beta_{n}=$ $\left.\beta_{0}+\lambda \cos (2 \pi n \chi)\right)$ at a frequency $\chi$ that is incommensurate with the frequency of the lattice (dashed line). 
exhibits a sharp transition, and becomes localized around a single lattice site. All the other eigenmodes of the AubryAndré model exhibit the same sharp transition, becoming tightly localized at different sites of the lattice above $\lambda / C=2$. For comparison we present the same calculation for a disordered system based on the Anderson model [1] [Fig. 1(b)], in which the term $\lambda \cos (2 \pi n \chi)$ in Eq. (1) is replaced with $\lambda W_{n}$, where $W_{n}$ are random numbers drawn from a flat distribution ranging from -1 to 1 . In this case there is no sharp localization transition at nonzero $\lambda$, instead there is a smooth decrease of the localization length as the strength of the disorder $\lambda$ is increased. For infinite one-dimensional lattices, all eigenmodes of the Anderson model are localized, even for arbitrarily small disorder strengths [2]. In a finite size system and weak enough disorder, the eigenstates appears to be extended since their localization length is larger than the system size. As disorder increases, the eigenmodes become localized within the finite lattice, with modes near the edges of the spectrum becoming localized first [2,19]. This, however, cannot be considered as a phase transition since the amount of disorder needed to observe localization goes to zero as the system's size goes to infinity. In contrast, in the AubryAndré model all eigenmodes are extended below the transition and become localized simultaneously above $\lambda_{c}=$ $2 C$, with a typical width smaller than the size of the system. The width of the critical region around $\lambda_{c}=2 C$ only weakly depends on the system size, and the transition is clearly visible for a system with 99 sites [Fig. 1(a)].

For a finite size system of length $L$, the requirement to be incommensurate is that no commensurate frequencies having more than one period within $L$ would be less than $1 / L$ apart from the incommensurate frequency ratio [25]. For $L=99$, the closest commensurate frequency ratio within $1 / L$ of the incommensurate ratio is 1.62 , with a period of 50 sites. If the incommensurability condition described above is not met, the system does not exhibit a sharp localization transition. For example, in a recent experiment, Roati et al. studied localization of noninteracting ultracold atoms [4] using a potential formed by two sine waves with a frequency ratio of $\sim 1.1972$. The closest commensurate frequency within $1 / L$ of the incommensurate ratio in this case was 1.2 , with a period of 5 sites. As a result, the passage to localization became smooth and localization was observed around $\lambda / C=7$ [26].

The sharp localization transition has a profound effect on the transport properties of the system [21]. Consider a spatially narrow initial wave packet confined to a single site. Such a wave packet will expand indefinitely if the eigenmodes of the system are extended, but will come to a halt if all the eigenmodes are localized [27]. Below the transition the expansion speed continuously decreases as $\lambda$ increases, settling to zero for $\lambda>\lambda_{c}$ [21]. Here we measure wave packet expansion to observe the signature of the localization transition in quasiperiodic lattices.

In the experiment we use a one-dimensional photonic lattice of evanescently coupled waveguides [28].
Structures of this type were recently used to study linear and nonlinear phenomena in one- and two-dimensional disordered lattices [17-19], featuring the observation of localized eigenmodes and exponential (Anderson) localization of expanding wave packets. Similarly, twodimensional photonic quasicrystals were realized in [29]. The important feature of these lattices is that the time evolution of waves inside the lattice is combined with propagation in another spatial dimension [30]. This way, it is possible to directly image the propagated wave function inside the lattice, in contrast to traditional transmission or conductance measurements. Another important advantage is the ability to introduce nonlinearity in a controlled manner and directly observe its effect on transport.

The propagation of light in waveguide lattices has been shown to be well described by the discrete nonlinear Schrödinger equation [28]. For an incommensurate photonic lattice, Eq. (1) will now read

$$
\begin{aligned}
i \frac{\partial \psi_{n}}{\partial z}+\left[\beta_{0}+\lambda \cos (2 \pi n \chi)\right] \psi_{n} & +C\left(\psi_{n-1}+\psi_{n+1}\right) \\
& +\gamma\left|\psi_{n}\right|^{2} \psi_{n}=0,
\end{aligned}
$$

where $z=c t$ is the free propagation axis, $c$ being the speed of light in the medium, $\beta_{0}$ is the single-site eigenvalue of the underlying periodic lattice, and $\gamma$ is the Kerr coefficient. In the linear limit $(\gamma=0)$ the equations are identical to the tight-binding model, while for nonzero nonlinearity these equations are also used to describe the mean-field evolution of a weakly interacting Bose-Einstein condensates in an optical lattice.

To realize the Aubry-André model, we fabricated a set of quasiperiodic waveguide lattices, with an incommensurate ratio of $\chi=(\sqrt{5}+1) / 2$ (the golden mean) and different values of $\lambda$ (see methods). To measure the expansion rate of wave packets, we injected a narrow beam into a single site at the input of the lattices, and measured the output intensity distributions. For each lattice we have repeated this procedure for 60 different sites in the same lattice and averaged the result. Identical samples with different lengths were used to measure the expansion rate of the wave packets. Results are presented in Fig. 2. Figure 2(a) depicts the expansion of a single-site wave packet in a periodic lattice $\lambda=0$ for two different times (equivalent to $6 \mathrm{~mm}$ and $21 \mathrm{~mm}$ of propagation). Figure 2(b) depicts the same results for an incommensurate lattice with $\lambda / C=$ 1.6. Here too the wave packets expand during the propagation, yet at a reduced speed as expected from theory $[21,27]$. When an incommensurate lattice with $\lambda / C=3.1$ is used [Fig. 2(c)], the wave packet remained tightly localized to the input site, and did not expand throughout the propagation.

The width of a wave packet can be characterized by the participation ratio (PR) of the wave function, given by $\mathrm{PR}=\left(\sum\left|\psi_{n}\right|^{2}\right)^{2} / \sum\left|\psi_{n}\right|^{4}$. A measure of the transition can be obtained by plotting $\langle\mathrm{PR}\rangle$ (averaged over initial positions) along the propagation for different values of $\lambda$. 

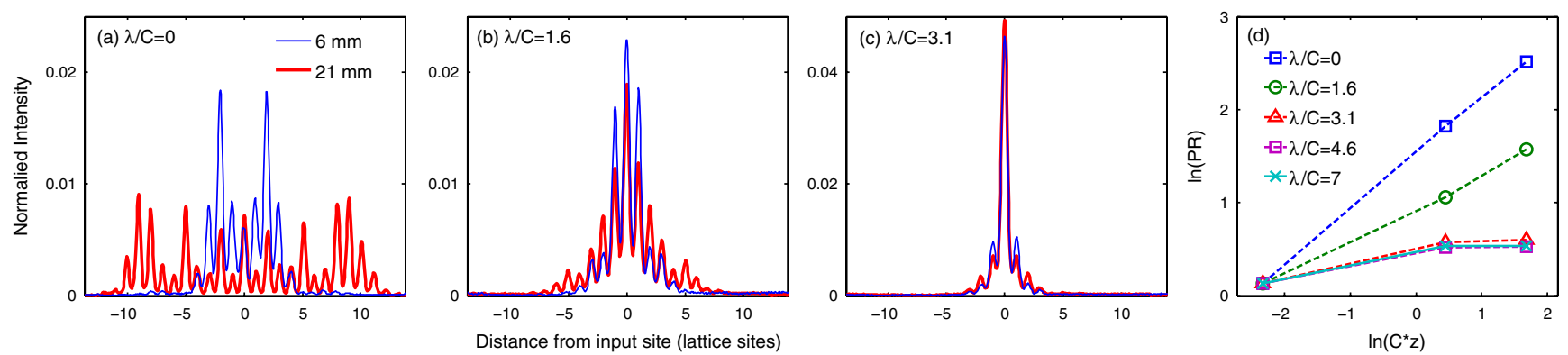

FIG. 2 (color online). Measurements of a localization transition in incommensurate photonic lattices. (a) The expansion of a singlesite initial wave packet in a perfectly ordered lattice $(\lambda=0)$ after $6 \mathrm{~mm}$ of propagation (blue) and $21 \mathrm{~mm}$ (red). (b) The same for an incommensurate lattice with $\lambda / C=1.6$. The wave packet still exhibits expansion, but at a reduced speed. (c) The same for an incommensurate lattice with $\lambda / C=3.1$. The wave packet remains tightly localized about the input position throughout the propagation, signifying localization. (d) Logarithmic plots of the averaged width of the wave packets (measured by the participation number PR) at different lengths and for different $\lambda$ values. These curves show the transition from expansion to localization as a function of the incommensurate modulation strength, signifying the localization transition.

The results are presented in Fig. 2(d), showing the transition from expansion to localization as a function of the modulation strength. The collapse of the curves for $\lambda / C>$ 2 in Fig. 2(d), as well as their saturation indicate the localization transition. To compare our results to theoretical predictions, we plot the measured $\langle\mathrm{PR}\rangle$ of the wave packets after $21 \mathrm{~mm}$ of propagation and the expected values calculated using Eq. (2) in Fig. 3(a). Figure 3(b) shows the calculated average width of the expanding wave packets as a function of the modulation ratio, for different propagation times. The curves show the transition becomes sharper for longer propagation times. The transitions is almost fully converged at $C z=5$, corresponding to the experiment.

Since the photonic lattices used in these experiments display nonlinear effects at high light intensities, they offer the possibility to study experimentally the effect of nonlinear interaction on the propagation before and after the transition. Various aspects of the role of nonlinear inter-
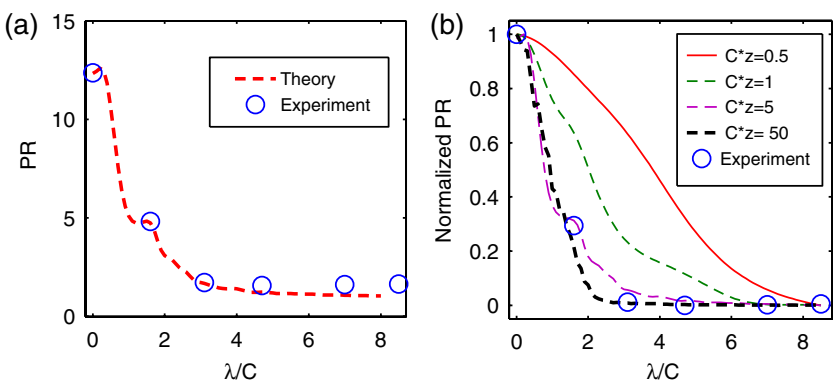

FIG. 3 (color online). (a) Comparison between the measured width of the wave packets after $21 \mathrm{~mm}$ of propagation (circles) and the theoretical values expected from Eq. (2) with $\gamma=0$ (line), for different values of $\lambda$. (b) Theoretical curves for the average width of the expanding wave packets as a function of the modulation ratio, for different propagation times. Each curve is normalized between 0 and 1 . The curves show the transition becomes sharper for longer propagation times and is almost fully converged at $C z=5$, corresponding to the experiment which is given on the same scale in blue circles. actions in incommensurate lattices were considered theoretically in [31-34]. An experimental investigation was made of BEC in quasiperiodic potentials [35], yet the strong interactions in this case are expected to result in a different quantum phase [36] which cannot be described by Eq. (2). The measured effect of nonlinearity on wave packet expansion in our system is presented in Fig. 4(a). Here we plot the averaged width of the wave packets after $21 \mathrm{~mm}$ of propagation as a function of nonlinearity, for different incommensurate modulation strengths $\lambda$. As the results show, for $\lambda=0$ an increased nonlinearity results in a reduced expansion of the wave packet. This is expected [28], since the sign of nonlinearity in our system corre-
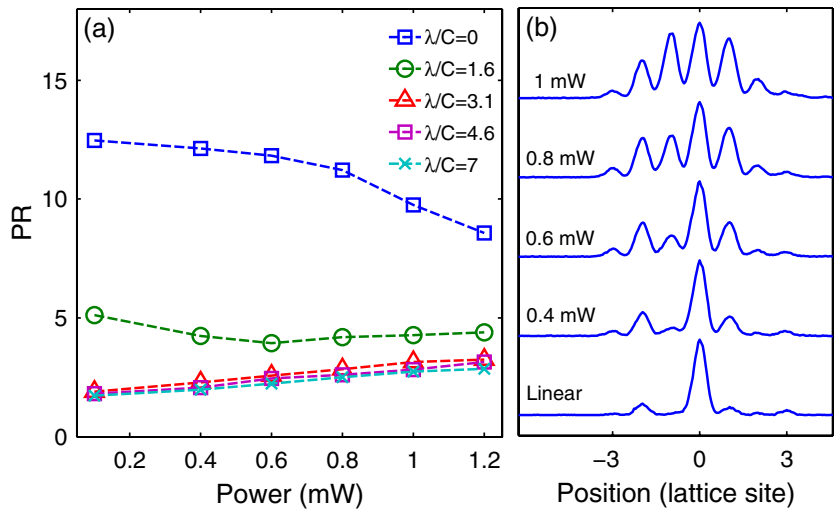

FIG. 4 (color online). Measurements of the effect of nonlinearity on wave packet expansion in incommensurate lattices. (a) The averaged participation ratios measured after $21 \mathrm{~mm}$ of propagation, as a function of power and for different incommensurate modulation depths $\lambda$. For $\lambda / C=0$, increased nonlinearity results in a reduced expansion of the wave packet. For $\lambda / C>2$ (the localized regime) nonlinearity results in weak expansion. (b) Measured output intensity distribution (horizontal axis) versus power for a single (nonaveraged) initial condition, in a lattice with $\lambda / C=3.1$. In the linear case the output is tightly localized to the input site, yet it spreads as the power is increased. 
sponds to self-focusing interaction. For $\lambda / C>2$ (the localized regime) we find that at some input positions, wave packets that are localized in the linear regime exhibit weak expansion under the influence of nonlinearity. A typical example is presented in Fig. 4(b) for a lattice with $\lambda / C=$ 3.1. This effect underlies the increase in the averaged participation ratios presented in Fig. 4(a) for $\lambda / C>2$, indicating a slight increase in width of the localized wave packet, induced by nonlinearity. For $\lambda / C=1.6$ (still below the localization transition) the expansion is reduced at low powers, and slowly recovers at higher powers. We note that the increase of the participation number with nonlinearity is reproduced by simulations based on Eq. (2) only at longer propagation distances. Possible mechanisms for this discrepancy are higher-order nonlinear effects or nonlinear absorption.

In conclusion, we have observed the signature of the Aubry-André localization transition by directly measuring the spread of initially narrow wave packets in maximally incommensurate photonic lattices. We found that in the localized regime, nonlinear effects result in a slight increase in the width of the localized wave packets. Possible extensions of this work involves the scrutiny of the critical regime around $\lambda / C=2$ and the study of the role of nonlinearity near the localization transition. Another open issue is the survival of localization in the presence of nonlinearity in the long time limit, a subject currently debated in relation to disordered (Anderson) lattices [37].

Methods.-To realize the Aubry-André model, a set of quasiperiodic waveguide lattices on an $\mathrm{AlGaAs}$ substrate was fabricated, using high resolution, large field $e$-beam lithography (Vistec VB6), followed by reactive ion etching. In designing the array to obtain the AA Hamiltonian we control the width of each waveguide that determines the on-site potential and the separation between neighboring waveguides that determines the nearest neighbors tunneling rate $C$. The width of each waveguide is designed to yield an incommensurate modulation according to Eq. (2) with $\chi$ being the golden mean. The average waveguide width and separation are $4.5 \mu \mathrm{m}$ and $5 \mu \mathrm{m}$, respectively. The waveguide separation was designed to yield an homogeneous tunneling rate across the array. The final values for the coupling rate $C$ was measured to be $250 \mathrm{~m}^{-1}$. Knowing the design profile of the sample and the coupling, we calculated the etch depth to be 1.3 microns, which we verified independently using a surface profiler (Zygo NewView 5000). Seven samples were fabricated, corresponding to different values of $\lambda$. The obtained modulations rates were calculated numerically from the design profile, and from the etch depth of the fabricated sample. The finite resolution of the fabrications process $-50 \mathrm{~nm}$, much smaller than the light wavelength, produced a small amount of on-site disorder in the actual Hamiltonian. Using numerically exact calculations based on the final fabricated profile we were able to estimate the strength of this fabrication-related disorder to be less than $0.05 C$. We verified that this amount of disorder has no significant effect on the localization transition, as the localization length associated with this disorder is much larger than our system.

We thank N. Bar-Gill for illuminating discussions. This research was supported by the Crown Center of Photonics, NSERC and CIPI (Canada) and EPRSC (UK).

*yoav.lahini@weizmann.ac.il

[1] P. W. Anderson, Phys. Rev. 109, 1492 (1958).

[2] B. Kramer and A. MacKinnon Rep. Prog. Phys. 56, 1469 (1993); P. A. Lee and T. V. Ramakrishnan, Rev. Mod. Phys. 57, 287 (1985).

[3] J. Billy et al., Nature (London) 453, 891 (2008).

[4] G. Roati et al., Nature (London) 453, 895 (2008).

[5] S. Fishman et al., Phys. Rev. Lett. 49, 509 (1982).

[6] F. L. Moore et al., Phys. Rev. Lett. 75, 4598 (1995).

[7] J. Chabe et al., Phys. Rev. Lett. 101, 255702 (2008).

[8] J. D. Maynard, Rev. Mod. Phys. 73, 401 (2001).

[9] P. Sheng Introduction to Wave Scattering, Localization, and Mesoscopic Phenomena (Springer, Berlin, 2006).

[10] O. Shapira and B. Fischer, J. Opt. Soc. Am. B 22, 2542 (2005).

[11] B. Fischer et al., Opt. Lett. 27, 1061 (2002).

[12] S. Longhi, Phys. Rev. A 77, 015807 (2008).

[13] A. A. Chabanov et al., Nature (London) 404, 850 (2000).

[14] D. S. Wiersma et al., Nature (London) 390, 671 (1997).

[15] M. Storzer et al., Phys. Rev. Lett. 96, 063904 (2006).

[16] C. M. Aegerter et al., J. Opt. Soc. Am. A 24, A23 (2007).

[17] T. Pertsch et al., Phys. Rev. Lett. 93, 053901 (2004).

[18] T. Schwartz et al., Nature (London) 446, 52 (2007).

[19] Y. Lahini et al., Phys. Rev. Lett. 100, 013906 (2008).

[20] S. Aubry and G. André, Ann. Isr. Phys. Soc. 3, 133 (1980).

[21] T. Geisel et al., Phys. Rev. Lett. 66, 1651 (1991).

[22] S. Ya. Jitomirskaya, Ann. Math. 150, 1159 (1999).

[23] C. Aulbach et al., New J. Phys. 6, 70 (2004).

[24] P. G. Harper, Proc. Phys. Soc. London Sect. A 68, 874 (1955).

[25] N. Bar-Gill et al., arXiv:cond-mat/0603513.

[26] M. Modugno, arXiv:0901.0210.

[27] See EPAPS Document No. E-PRLTAO-103-031928 for a comparison between theory and experiment for expanding wave packets. For more information on EPAPS, see http:// www.aip.org/pubservs/epaps.html.

[28] D. N. Christodoulides, F. Lederer, and Y. Silberberg, Nature (London) 424, 817 (2003).

[29] B. Freedman et al., Nature (London) 440, 1166 (2006).

[30] H. De Raedt et al., Phys. Rev. Lett. 62, 47 (1989).

[31] M. Johansson et al., Phys. Rev. B 52, 231 (1995).

[32] G. Kopidakis et al., Europhys. Lett. 33, 459 (1996).

[33] G. S. Ng and T. Kottos, Phys. Rev. B 75, 205120 (2007).

[34] A. A. Sukhorukov, Phys. Rev. Lett. 96, 113902 (2006).

[35] L. Fallani et al., Phys. Rev. Lett. 98, 130404 (2007).

[36] M.P. A. Fisher et al., Phys. Rev. B 40, 546 (1989).

[37] G. Kopidakis et al., Phys. Rev. Lett. 100, 084103 (2008); A. S. Pikovsky and D. L. Shepelyansky, Phys. Rev. Lett. 100, 094101 (2008); I. Garcia-Mata and D.L. Shepelyansky, Phys. Rev. E 79, 026205 (2009); S. Flach et al., Phys. Rev. Lett. 102, 024101 (2009).S. Fishman et al., Phys. Rev. E 78, 066605 (2008). 